\title{
Systemically Integrated Approaches to Coaching: An Introduction
}

\author{
Sean O'Connor \\ Coaching Psychology Unit \\ University of Sydney \\ Sydney, Australia
}

\begin{abstract}
Coaching approaches attempting to support leadership development often occur in isolation and lack integration into more wholistic approaches to change and development. Systemic coaching approaches promise much of what could be possible, however these systemic approaches are still largely undefined. The concept of systemically integrated approaches to coaching is introduced and explored here to provide utility to coaches working with leaders in organisations. Complex Adaptive Systems theory and Complex Responsive Processes are presented briefly as theoretical frameworks in which to apply Network methodologies and Network Thinking for the benefit of individuals, groups, teams and organisations. In this paper, systemic approaches to coaching include coaching through a systemic lens, coaching to enhance systemic thinking and systemic competency, coaching for systemic change and, finally, an integrated approach to systemic coaching. It is hoped that, as an industry, we might begin to think more carefully about what it could really mean to coach systemically and the potential benefits for leaders and organisations of a truly systemically integrated approach.

Keywords: Coaching, Systemic Coaching, Network Cognition, Systems Theory, Organisational Change, Social Network Analysis.
\end{abstract}

\section{Introduction}

As society, commerce and politics become far more interconnected, interrelated and complex, the need for more effective approaches to adaptation and change seem paramount. The coaching industry, if it is to maintain relevance, needs to evolve in line with these global shifts in perspective and organisational needs. While coaching research continues to grow (Grant \& O'Connor, 2019) and more organisations utilise coaching services (Mann, 2019; ICF \& HCI, 2019), there is more that coaches could be doing to assist organisations adapt, transform and innovate in response to their challenges. As coaching interventions have become more complex, they have been hampered by a lack

This is an Open Access article distributed under the terms of the Creative Commons Attribution (CC BY) License which permits use, distribution and reproduction in any medium, provided the original work is properly cited. 
of clarity about their purpose, promotions laden with confusing terminology and a notable absence of empirical support. As such, key aspects of systemic coaching need to be more specifically articulated.

According to Bachkirova and Borrington (2020), in recent years coaching has become pre-occupied with some 'beautiful ideas', such as mindfulness, positive psychology and transformational coaching. Whilst the same might be said of systemic coaching, a substantial literature exist in respect of systems theory (Mele, Pels \& Polese, 2010), systems thinking (Arnold \& Wade, 2015) and systemic change within organisational contexts (Bunker \& Alban, 2012). Despite this, relatively little theoretical and empirical work that been reported on systemic approaches to coaching, despite the fact that it has gained an increasing amount of attention within the coaching industry (Lawrence, 2019). This is problematic from an applied perspective, as a lack of robust scholarship threatens to hinder the potential utility of systemic coaching for the development of both individuals and organisations.

The main aim of this paper is to introduce the idea of systemically integrated approaches to coaching. It begins with a a brief overview of Complex Adaptive Systems theory and Complex Responsive Processes (Stacey, 2001, 2012a), and their relevance to leadership and organizations, and to coaching. This is followed by a review of related theory and research on networks, social network analysis and the "coaching ripple effect" (O'Connor $\&$ Cavanagh, 2013). Concepts of systemic and network thinking are also explored, along with a review of the importance of context. The paper concludes with a discussion of systemically integrated approaches to coaching at various systemic levels. While many of the ideas presented here require further investigation and, deserve greater attention that is possible in this paper, they are offered as a starting point for interested researchers and practitioners. More broadly, it is hoped the coaching industry might begin to think more carefully about what it means to call oneself a "systemic coach", and what the potential benefits might be of utilizing a systemically integrated approach to coaching and development.

\section{Clarifying Concepts: Complex Adaptive Systems and Complex Responsive Processes}

Theoretical approaches to understanding systems have developed and evolved over time to include many different perspectives. These include earlier more mechanistic and reductionist views such as cybernetics (Weiner, 1948), and general systems theory (Von Bertalanffy, 1972), to more complex and non- 
linear articulations such as complexity and complex adaptive systems theory (Holland, 2006, Dooley, 1997), as well as particular theories related to human interaction in systems such as Stacey's complex responsive processes (Stacey, $2001 ; 2012 a)$.

Given that coaching is essentially a developmental approach based on (mostly dyadic) interactions, Complex Adaptive System (CAS) and related theories are of particular interest as they provide a theoretical framework for understanding the behaviour of human systems through the lenses of interaction and interconnectivity. From a CAS perspective, organisations emerge from a diverse network of interacting components that grow and adapt in response to interaction between these components as well as change in the internal and external environment (Eidelson, 1997). According to this view, large complex hierarchically ordered systems emerge from the interaction of local system components (Stacey, 2001; 2012a; Stacey \& Mowles, 2016). System adaptation is also seen as novel, with system components interacting through their own agency, providing feedback to the system impacting on ongoing, interaction, behaviour and change (Cavanagh, 2006). The impact feedback has on change is often non-linear and iterative (Cavanagh \& Lane, 2012) making specific shortterm prediction difficult if not impossible (Stacey, 2012b). This also leads to many of the features of a system such as the growth, culture, climate and felt experience being thought of as emergent (Stacey \& Mowles, 2016).

While Stacey and Mowles (2016) argue for specific differences between systems generally and human interaction, Complex Responsive Processes (CRP) are important to acknowledge because they emphasize the interaction of individuals (agents) locally and the contribution of that local interaction to human interactive evolution as well as the relationship to emergent phenomenon more broadly. Whilst there is much to debate on the nuanced differences between CAS and CRP, it is sufficient here to note that they differ in the view they take about the role of agency and the extent to which human agency requires special consideration. Irrespective of this point of debate, CAS has value for understanding systems that include human agency, while CRP provides insight into human interactions that occur amid complexity in more nuanced and useful ways.

CAS theory helps us to understand interactions between components of a system, and how change in one aspect of a system can influence change in other aspects of the same system (Dooley, 1997; Eidelson, 1997). It suggests that the interaction and the quality of that interaction are important for the emergence of self-organisation (Stacey, 2001; 2012a) and adaptation, which are 
important for a system's growth and survival (Fredrickson, 2000). This suggests organisations are a complex web of interactions and relationships with emergent properties that reveal themselves over time. From a practical standpoint, understanding systems as interactive, interconnected networks impacts upon how change management is attempted and how leaders' function and develop as influential and embedded agents in these networks of interaction.

Given the complex, difficult and unpredictable nature of many contemporary challenges (Rammel, et al., 2007), perspectives like CAS and CRP provide frameworks for addressing these challenges, through the development of individuals, groups and organisations. CAS theory can be seen as an important way to conceptualise organisations, with a great deal of potential for supporting leadership development within those organisation. CAS and CRP suggest that interconnectivity and quality of interaction, in particular local interaction (Stacey, 2001; 2012a), are key supporting development and, thus represent a useful focal point for coaching engagements. Indeed, CAS and CRP can be beneficially explored in leadership to provide perspective and create insight into systemic emergence and the dynamics of interaction in a system. This can be useful for helping leaders better understand their experiences, by viewing the dynamics of interactions and behaviour differently and, hopefully, find different ways to purposefully shape their own behaviour (particularly, as emphasised by CRP, within their local networks of interaction).

A major criticism of both CAS and CRP is that they are notoriously difficult to operationalise for both research and practice purposes (Brown, 2006; Houchin \& Maclean, 2005; O’Connor \& Cavanagh, 2013). This difficulty operationalising the concepts diminishes their utility to what can be gained from metaphor and reflective, post-hoc interpretations of action, with little predictive benefit within the psychological, organisational and social sciences. However, the network science and related methodological approaches (such as social network analysis) do offer some promise as ways of operationalising CAS and CRP. See Table 1 for a summary of these concepts, and others to be discussed in this paper.

\section{Networks, Social Network Analysis and the Coaching Ripple Effect}

CAS and CRP both identify the importance of interconnectivity and interaction, which aligns them to the view that organisations are networks of interaction and relationship (Schneider \& Somers, 2006). Network science is a broad field of scholarship concerned with the analysis and understanding of all 
types of networks, both static and dynamic. Where they differ from existing linear-based approaches in understanding groups and systems is through their focus on relational data, which is defined as that which travels, transfers or moves between individual nodes or system components. Relational data includes, but is not limited to, constructs such as the spread of disease, perceptions of friendship, trust, and interaction (Scott, 2000). According to this view, an organisational network can be made up of the individual members of an organisation and the different types of possible relationships between them (Galaskiewicz, 2007). Network science and related approaches are more concerned with what transmits between the individuals in the network, rather than the individuals themselves. As such, its focus is holistic and concerned with the broader pattern of connections amid these exchanges.

In this way network methodologies, such as social network analysis, function at a higher level of analysis than traditional reductionist approaches and, as has been argued, they operationalise CAS for the purpose of measurement (O’Connor \& Cavanagh, 2013). While network science and network approaches do not necessarily imply dynamic, changeable, selforganising patterns of interaction (which are important to both CAS and CRP), they also do they exclude them. Instead, the theoretical view of networks as entities of relational data is maintained, whilst permitting different forms of statistical analysis, including calculations that can reflect dynamic changes occuring in systems as the result of relationships and emergence.

Social network analysis (SNA; Scott, 2000) is a methodology suited to specifically examining networks or systems of relationship at multiple levels. By using SNA, researchers and practitioners can focus at a whole-of-network level (more holistic) and/or at the level of smaller connected subsystems (more local). Using relational data in a network, it allows for an examination of individuals within an embedded context, whilst taking multiple network connections and relationships into consideration. In doing so, it allows researchers to examine questions related to emergent properties, change over time, and interconnectivity (Scott, 2000). In line with CAS and CRP (Anderson, 1999; Bradbury \& Lichtenstein, 2000; Dooley \& Lichtenstein, 2008, Stacey, 2012a), this mathematical approach to the macro and micro aspects of systems helps to illustrate the complexity and interconnectedness of systems, whilst allowing statistical analysis at multiple levels, including the dyadic, the ego network, or any identified groups or subnetworks (Borgatti, Everet \& Freeman, 2014). 


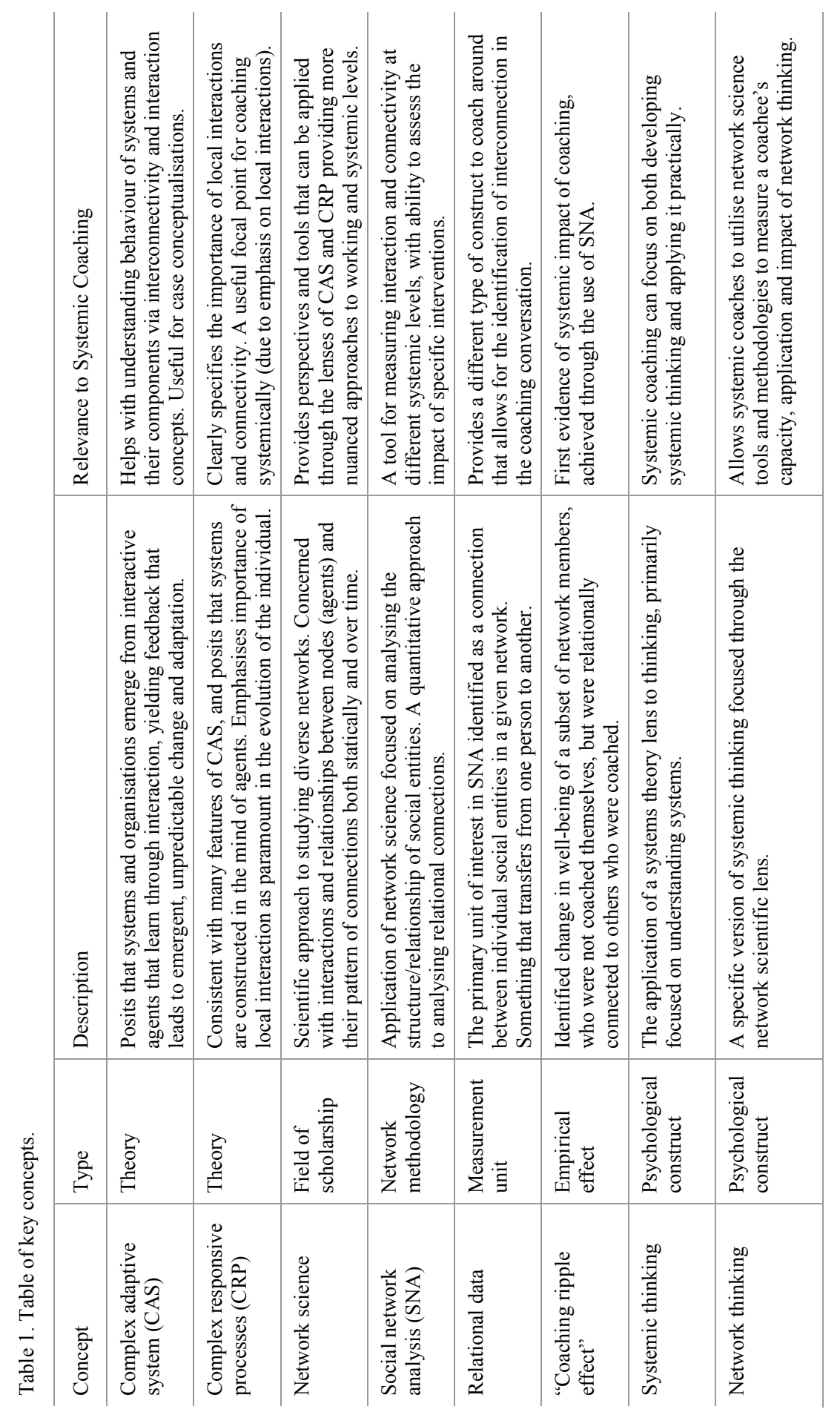


SNA has been employed with some success in researching the interconnected nature of leadership interaction and behaviour. Leadership research that has utilised SNA include investigations of group performance and leader reputation (Mehra, Dixon, Brass, \& Robertson, 2006), leadership distribution in teams (Mehra, Smith, Dixon, \& Robertson, 2006), shared leadership (Novoselich \& Knight, 2018), transformational leadership, group interaction and organisational climate (Zohar \& Tenne-Gazit, 2008), the advice and influence networks of transformational leaders (Bono \& Anderson, 2005), social capital in intra-firm networks (Tsai \& Ghoshal, 1998), and collaborative learning (Xie et al,. 2018; Sineema et al., 2020). More applied uses include SNA as a leadership diagnostic tool for team coaching (Terblanche \& Erasmus, 2018).

Within coaching research, SNA has helped to reveal the existence of a "ripple effect" in coaching (O'Connor \& Cavanagh, 2013). This research was the first to use a systemic approach to measurement to examine the impact of coaching on leaders beyond those who were coached directly. Underpinned by CAS and CRP, this study helped to identify how coaching-induced change might radiate from coached leaders to others who were connected through the quality of their interactions. While a full review of this research is beyond the scope of this paper, a key finding was that the pattern of the network mattered for how wellbeing shifted across the organisation. The closer individuals were embedded in the interaction network of the coached leaders (i.e., the more connected they) the more likely their psychological wellbeing was to positively improve. This supported arguments previously advanced by Stacey about CRP $(2001 ; 2012 a)$, that changes in the local interaction are important for the development and experience of the local interconnected network. In another interesting finding, changes in leader behaviours appeared to be met with some resistance by those they were connected to (as reflected in slight decreases in perceived relationship quality) even though these changes were also observed to increase their wellbeing.

These findings represent the first pieces of evidence that coaching cultures might be created through solution-focused, cognitive-behavioural coaching and that leadership change can potentially ripple or spread through the interaction quality of an organisation. This is important because it identifies a much broader impact for coaching beyond the coached individual, and supports the conception of systems through CAS and CRP lenses. This study has also raised some controversial questions. For example, who is a coach coaching for? Is it only, as has long been assumed, for the person sitting directly in front of the coach? This raises additional ethical questions, in regard to the impact and 
effect of coaching. It suggests that the coach has a duty of care to the greater organisation and to the individuals connected to the coachee. Whilst the assumption of limited responsibility (to the direct coachee) might be reassuring for coaches, this is hard to maintain for a coach who endorses an evidencebased approach.

\section{Systemic and Network thinking}

It should also be noted that the interconnected influence of coaching engagements raises exciting possibilities about how a coach might engage a system to support adaptive change at multiple levels of that system. How can coaches think differently so that they might create more targeted and effective interventions? How do they need to understand systems, help leaders understand systems, and engage with (and in) systems? In the next section, these questions will be considered via an overview of two related (though poorly understood) concepts: systemic and network thinking.

\section{Systemic thinking}

To date the idea of systemic coaching is largely associated with the idea of systemic thinking. However, little has been done to define the potential utility and the varied ways that systemic approaches through systemic thinking may be employed for the benefit of positive change, particularly through leadership coaching. Although a full review of the varied definitions and perspectives of systemic thinking is not possible here (see Lawrence, 2019 for a recent overview relevant to coaching), systemic thinking can be thought of as the application of a systems theory lens through which to view the world (Arnold \& Wade, 2015). From a CAS or CRP perspective, systems thinking is generally defined as a form of thinking that draws insight from the interrelationship between components of a system, rather than focusing on individual or dyadic components.

Lawrence (2019) argues that the particular systems theory a coach adopts may influence how they work with their coachees. Specifically, Lawrence discusses the following systemic perspectives. First-order systemic perspectives, which are reductionist in nature and assume that organisations can be broken down to their components and rules in order to discern organisational behavior. In contrast, second-order perspectives view systems as less discernable and predictable, while still holding to the assumption that one can stand 'outside' the system to observe and understand its behavior. The thirdorder perspective sees systems as complex, with an understanding of them 
focused on exploring interactions whereby agents interact to create emergent phenomenon. Finally, complex responsive processes are primarily based on the idea that organisational systems are not real and only emerge from the social interaction and responsiveness of the agents within a wider pattern of social interactions. This is a perspective that is not entirely inconsistent with many interpretations of CAS and could be interpreted as a specific case of complex systems.

Any coach who defines themselves as being "systemic", may do so in order to explain that their coaching focuses on developing the systemic thinking of coachees. An implication of Lawrence's (2019) discussion is that coaches should make their theoretical position on systems explicit, if they wish to adequately define their practice. However, given that coaches are likely to be informed by different theoretical positions, there is bound to be confusion regarding the type of thinking a particular coach is aiming to develop in a coachee. This would be particularly so when the terms "systemic coach" and "systemic thinking" are used without clarification or specific definition. Coaches focusing on developing thinking, in lieu of labeling themselves as systemic coaches, should be identifying the type of systemic thinking they seek to develop in their clients. This could help to specify particular coaching approaches relevant for particular organisations and their leadership development requirements. What might work best in a given organisation may depend on the organisations current level of complexity, or the developmental stage of a leader the coach may be preparing to work with. Not all organisations need their leaders to be complex adaptive systems thinkers, and leaders may not need to engage with complex adaptive conceptions of organisational behavior in order to grow and develop. Indeed, many leaders struggle to apply CAS and CRP ideas in practice.

Developing systemic thinking in leaders may be attractive to organisations for a number of reasons. As many organisations struggle with the seemingly increasingly volatility and uncertainty of the complex operating environments, having leaders with an increased systemic thinking capacity would seem beneficial. It is assumed, for example, that being able to understand the interaction and relationship between disparate components of an organisation and its environment, provides opportunities for creativity, innovation and enhanced performance. However, without clearer definitions and articulated approaches, assisting organisations in this way will continue to be difficult. 


\section{Network Thinking}

A specific approach to developing systemic thinking is to work directly with leaders from a network perspective, and develop network thinking. This is important because of the utility identified earlier in network-based approaches to operationalise some of the concepts of CAS and CRP. Network thinking here is a specific version of systemic thinking that clearly articulates its theoretical connection to network science. Further, there are practical approaches used for measurement (i.e., social network analysis) within the field that can be applied to network thinking in order to assess capability to think through a network lens.

While there is still very little research, there exists evidence supporting the potential for developing network thinking in leadership. This is mostly defined in the cognitive network structures or network cognitions literature. According to Krackhardt (1987), network cognition refers to mental maps and connections that individuals hold about the relationships between individuals and/or other system components (such as teams, customers or any identified agent group). If you ask someone to identify who trusts who, or who has worked with who, in an organisation or team they can usually draw a map of connections. This represents the network cognition they hold based on the relational data identified in the question asked. Networks have been identified as important for leaders for a number of reasons. These include information exchange, flow and diversity (Balkundi \& Kilduff, 2005), reputation, influence and support, team performance (Balkundi \& Harrison, 2006) opportunity recognition (Kilduff \& Tsai, 2003), change management, as wells as innovation and creativity (Rodan, 2010; Freidrich et al., 2011, O'Connor \& Cavanagh, 2013).

Another important concept related to leadership performance is researched within the field of network cognitions is cognitive accuracy. The accuracy of network cognitions is represented by the degree to which a network cognition correlates with the actual network that exists when measured objectively (usually assessed using SNA). Leaders with more accurate network cognitions are rated by others as more powerful and influential, and tend to be higher performers (Krackhardt, 1990). There is also evidence of common network-level cognitive distortions, whereby individuals tend to see themselves as closer to the center of a network. In addition, it is also common that relationship perceptions of ourselves to others will be reciprocated (Casciaro, 1998; Kilduff \& Krackhardt, 2008). That is, we assume that if we are connected to two people then those two people are also connected. Given the potential 
value of cognitive accuracy to a leader, there would appear to be some value in applying cognitive-behavioural techniques to the development of network thinking (Grant, 2013).

Based on the evidence reviewed above regarding the coaching ripple effect (O'Connor \& Cavanagh, 2013), the use of coaching to develop network thinking in leaders may have utility for leadership development in organisations. Anecdotally, leaders are often easily able to grasp the idea of networks of connections that exist in an organisation or team, allowing coaches to work directly with mapping such connections, and identifying evidence for their existence through direct observation and testing. Gathering evidence to assess, test and adjust network cognitions can work to enhance network cognition accuracy and, in turn, provide direct evidence of a change to more systemic thinking. Such a change is consistent with the features of CAS and CRP identified earlier in this paper, and directly ties CAS and CRP (through network thinking) to specific actions that may benefit not only the leader, but also the team, group or organisation within which the leader is embedded.

Increasing a leader's capacity and aptitude for network thinking scaffolds them towards complexity-based systems thinking by enabling them with increased accurate perceptions of local connectivity in their system, allowing for the inclusion of interactive dynamics in decision making and engagement. While this does not infer predictive knowledge of the system over time (which would be inconsistent with CAS and CRP), it does infer the potential for reflective and adaptive engagement with the system as it shifts and responds emergently to these very same engagements. While this proposition and line of argument requires further inquiry and specific empirical research, the underpinning evidence seems to support the potential for success here.

At this point it is important to note that the process identified here of increasing network or systemic thinking through coaching, occurs through the process of interaction, influencing the experience of individuals connected to a leader, who are all interacting within a given context. In fact, all development occurs within the given context of an individual. We now turn to discussion on the relation between leadership, context and development more specifically.

\section{The Importance of Context for Development}

Individual and leadership development occurs within a particular interactive environment. These environments are represented by where and how individuals work and the processes, practices, policies and procedures of 
working that emerge as part of the organisational function and evolved interaction of local connected groups. Despite the obvious importance of context and its potential impact on individual and organisational growth, surprisingly little research has been reported on the impact of emotional, psychological and physical environments on development.

A recent literature review focused on positive psychology and buildings and workplace communities sought to identify how the interactive and physical context of a workplace might relate to wellbeing and performance (O'Connor \& Grant, 2018; Grant, O’Connor \& Studholme, 2019). According to the authors, the quality of the physical environment is an important factor, with factors such as air quality influencing performance on cognitive tasks (Allen et al., 2016), and impacting respiratory health (MacNaughton et al., 2015). These observations align with those of Gensler (2005), who identified that office design contributed to a $19 \%$ increase in productivity, and others who have identified strong links between sunlight, views and air conditioning with productivity and positive workplace behaviour (Mulville, Callaghan \& Isaac, 2016). Psychologically, this review reported that high performance workplaces are known to have strong culture, values and social contracts with staff, and that these are linked to higher levels of engagement (van Elst \&Meurs, 2015), productivity and performance (Guthrie, 2001), and lower intension to leave (Bakker, 2011). It has also been identified that staff in these work places generally hold themselves and their organisations in higher esteem (Cameron \& Dutton, 2003; Pierce, Gardner, \& Crowley, 2016) and that positive leadership is highly beneficial (Jones et al., 2016; Cameron, 2012; Grant, 2013; O’Connor \& Cavanagh, 2013; Datta, 2015).

This review reinforced previous work on the importance of organisational context by Cameron (2012), which emphasised positive organisational climate, positive relationships, positive communication and positive meaning at work. A fifth factor of the positive built workplace environment (PBWE) was defined as an environment "designed and operated in a way that provides the optimal physical and psychological conditions and resources to enable employees to consistently deliver high performance and maintain personal and organisational well-being." (Grant, O'Connor \& Studholme, 2019, p. 71). In an illustrative case study, O’Connor \& Grant (2018) reported that a PBWE was also important for supporting the three basic psychological needs (i.e., autonomy, competence and relatedness) specified in self-determination theory (Deci \& Ryan, 2000). This is noteworthy because these needs are known to support optimal functioning and well-being (Spence \& Deci, 2013; Spence \& Oades, 2011), at both the individual and organisational level (Deci, Olafsen \& Ryan, 2017; 
Gagné \& Deci, 2005). Further to this, Grant, O’Connor and Studholme's (2019) qualitative analysis identified additional themes regarding the importance of leadership and interactions at all organisational levels, how organisations and leaders utilised the environment to facilitate significant positive cultural, organisational, and individual change, and how positive emotions were supportive of adaptation. These findings provide some support for the connection between workplace community members and physical environments, as they pertain to performance, wellbeing, leadership and development.

\section{Discussion}

So far, CAS and CRP have been identified as important theoretical conceptions of organisations that emphasis the importance of interconnectivity and interaction. Network science has added a view of connectivity and the dynamics of networks that is not inconsistent with either. SNA has also been reviewed to provide an understanding of how systems concepts can be operationalised, and permit the measurement of dynamics in a system. As described, such concepts and methods can support developmental processes (supported by coaching) that use network perspectives to enhance network thinking and systemic thinking in leaders. Additionally, a review of the coaching ripple effect has identified how coaching can create impacts beyond individuals coached in an organisation, through the direct application of a network-based approach with the results aligning with theory presented by CAS and CRP. Further to this, qualitative research at the organisational level suggests that workplace community and the actual physical environment may very well support personal wellbeing and development in important ways, and that the interactive environments leaders create during their own development may support personal growth and development of others in the system.

All of this suggests that coaching can have an additional impact at the systemic level on the leader through cognition development, which can have an effect on behaviour and dynamics (through interactions) at the betweenindividual level of the system, creating environments that impact the work experience (and development) of others. Such experiences are akin to a contagion that can ripple through a system in such a way that its aggregation may influence not only the physical environment but also the culture and climate of an organisation. So, if coaching can have an impact either directly or indirectly at multiple systemic levels, what does this mean for the notion of "systemic coaching"? 
The answer to this question needs to apply not only to how coaches think about systems (i.e., coaching through a systemic lens), but also for how leaders think in these systems, and therefore how coaches can help leaders develop this type of thinking (i.e., coaching for developing systemic competency and systemic thinking). Relevance is also required to how we work with leaders with the system or, as it is termed here, coaching with the system in mind. Finally, how we work with the system for the leaders and the organisation, or how we use aspects of the system to support leadership change and growth, an approach identified as systemically integrated coaching. We turn now to exploring these four alternative systemic coaching approaches more specifically.

\section{Coaching through a systemic lens}

One way to think of systemic coaching based on the empirical and conceptual review presented thus far is to think about the approach a coach might take in working with a coachee. She or he might use systemic concepts themselves, utilising systemic thinking and approaches to their questions that leverage the system in order to support change for the client. While the emphasis in this paper has been on CAS and CRP, other systems theories may be just as relevant in a given situation. From this view, the systemic thinking is a feature of the coach's skill set and may form the main lens through which they perceive the coachees goals and challenges. This may involve a coach asking the coachee questions about interactions, dynamics and relatedness processes that are systemic, or asking questions based on some feature of a systems theory (in the hope that it may shift their perspective on a given goal or challenge and reveal a potential solution or new path forward. Here the development of systemic thinking in the coachee is not a primary goal, and may or may not occur. Here systems theory and systems thinking are tools the coach uses to aid their own thinking, build a case conceptualization, or perhaps design an intervention in collaboration with their coachee. The approach a coach takes here is very likely to be influenced by the particular systemic perspective they are familiar with, or simply chooses to apply based on the relevance to the situation.

\section{Coaching for systemic competency and the enhancement of systemic thinking}

In order to develop systemic thinking in leaders and systemic competency in leadership, the goal of developing systemic thought in a coachee needs to be much more explicit. This is more likely to occur in a developmental coaching engagement where the identified challenge or goal is beyond the capability of 
the coachee at their current stage of adult development, or through the world view they currently hold around systems. Here the coach might be more likely to case conceptualise using systems theories as models for collaboratively understanding the dynamics of a system or organisation with the coachee they are working with. Psychoeducational might be useful here, as might specific application of network approaches to identifying structure in an organisation, group or team. Measurements of network cognition accuracy can also be used to identify shifts in a coachee's systemic thinking, along with measures of the systemic nature of solutions identified in session by the coachee. For example, identifying the degree to which a coachee looks first to the dynamics between aspects of a situation, as opposed to seeing problems as embedded in individuals.

When coaching for systemic competency, systemic thinking and network cognitions are much more an explicit goal of the coaching engagement. Coaching sessions are more likely to involve metacognitive discussions, providing a space where coachees can be scaffolded in applying multiple systemic lenses to identified goals or interpreting organisational experiences. In this way the coaching session becomes the training ground for thinking differently. If systemic competency is a goal, then the thinking must not be the only thing that changes. Change must also be seen in the actions that coachees take in the system and the degree to which these actions are informed by systemic thinking. Coachees should be increasing their capacity to hold more of the dynamics of a given organisational situation in mind when making decisions both in preparation and planning, as well as in real time. Coaches need to be thinking about how to develop activities and interventions that allow the coachee to apply systemic thought in shaping their behaviour in the organisation within which they work.

\section{Coaching with the system in mind}

Many of the challenges faced by leaders in organisations often centre around change and adaptation. Organisation growth and change often require dealing with tension and working with disparate parts of the system, as well as other organisations or systems that might be connected to the organisation in which a leader leads. In order to navigate the complexity of the required changes or need for adaptation, leaders are often required to bring a level of systemic thinking to bare on the approaches they take to engaging with change in the system. Leadership from this perspective is often about creating systemic changes in the organisation. While the above discussion on coaching for 
increased systemic thinking and systemic competency is certainly necessary for creating systemic change in an organisation, it may not be sufficient.

The additional component required when creating a systemic organisational shift is an explicit and agreed goal around organisational change. Through this a coach can then collaborate with a coachee in order to support the identification of CAS, CRP or other systems theory features as well as networkbased opportunities that can be leveraged to support the adaptation of the system in a potential direction. What is required here of both the coach and coachee is a high level of systemic and network thinking, a shared understanding of systems based organisational lenses and a high level of systemic competency. Whilst this approach can use all of the previous tools and coaching processes discussed, it is much more experimental. The coach and coachee need to engage with the goal of creating change in the system, monitoring system shifts and adapting approaches as emergence occurs. Coach and coachees are more likely to collaborate on the design of safe-to-fail experiments that allow identification of the systemic sensitivities in the current state of the organisation. These experiments may then allow for more complex organisational engagement strategies that can support appropriate intervention, adaptation and the potential for positive change.

\section{Systemic integrated coaching}

An integrated approach to systemic coaching combines the above approaches and seeks to engage a system a more direct way. This includes integrating change into the system within which the coachee is embedded, along with thinking about how to engage the system in a systemic way to support coach, coachee, and organisational development. As the support coaches provide becomes more complex adaptive, the relationship coaches create with leaders and their organisations can become much more collaborative and innovative. Coaches can engage with organisations to assist them in fulfilling their goals, while also creating an opportunity for important systemic shifts in how organisations seek to support wellbeing, performance and the development of employees at all levels.

Complexity theories, such as CAS and CRP, highlight the importance of relationship and interaction. Systemic and network thinking helps to shape the way specific organisations are viewed and understood. SNA and network approaches help identify practical strategies for working systemically, and research findings derived from such methods (e.g. O'Connor \& Cavanagh, 2013) provide confirmation that interactions ripple through networks in ways 
that can spread change and influence wellbeing. Additionally, the psychological and emotional space created by leaders impacts the developmental opportunities for employees and that certain qualities of environmental spaces also support performance, wellbeing and growth. Given all of this, how can "systemic coaches" work systemically to support flourishing for individuals, relationships, groups, teams and entire systems?

A systemically integrate approach to systemic coaching means using all that we now know as coaches to effectively engage systemically for change. Coaching begins not from the first conversation with a coachee, but from a coach's initial connection with the organisation. Every point of interaction is a connection in the network of relationships, which means a coaching philosophy can infuse every part of the engagement, from the contracting and engagement process through to coaching review. This philosophy can inform how coaches engage and has the potential to provide evidence of efficacy for the benefits of coaching as a form of interaction and the process of coaching for effective change.

Many coaching organisations are engaging businesses to provide support beyond simple individual coaching, and are coaching across leadership groups, as well as providing training and other consultative services. The design and development of the overall program of change is an opportunity to systemically integrate a program of development for the benefit of the business. Identifying oneself as a systemic coach means thinking about how to engage the system systemically. How are the coaching goals supported and encourage by the existing connections in the system? How do the current policies practice and processes in the organisation support or hinder a coaching approach or intervention? What measures and developmental activities exist in the organisation that might be integrated with, and enhanced by the coaching program? What can be done to engage with the system in a way that can shift systemic components towards supporting the change the organisation wants to see? These are just but a few questions systemic coaches can be asking to shape their engagement with the systems they work with, be it an individual coachee, a team, an organisation or the broader coaching industry.

For example, at the individual coaching level, systemically integrated coaching might include utilising measurement to inform the system of change to be expected in a coachee through the coaching engagement. This could be 360-degree feedback that is curated to ask questions regarding behaviours that are specific to a coachee's development plan or stated goal(s). If that feedback is sought from individuals in structurally important and/or influential positions 
in the interaction network of the organisation, then the questionnaire may prime those individuals to focus on the behaviours the coachee is attempting to work on. This inadvertently creates a feedback loop in the system that can then be utilised to positively reinforce change in leadership behaviour. Importantly, such a process could create a behaviour change expectation amongst 360 raters, such that they are less likely to resist the such change when they encounter it.

The above is a simple example of systemic coaching at the level of the individual, characterized by the systemic thinking inherent in the design of the feedback process. This process leverages the interaction network and the system itself to reinforce change in the coachee, whilst changing the expectations of the system and, therefore, changing the system itself. This is what systemic coaching could and should look like if the aim is to effectively create cultures of coaching to support organisations and leadership in meeting the challenging needs of the modern world.

\section{Conclusion}

This paper has introduced the concept of systemically integrated approaches to coaching by presenting related areas of theory and research that are relevant to systemic coaching. It aimed to present a more detailed articulation of what is means to be a "systemic coach" and has presented ideas that will hopefully scaffold debate about the potential positive impact that evidence-based approaches to systemic coaching might have for leaders, teams, organisations, and society more broadly. Whether one is (i) coaching through a systemic lens, (ii) coaching for systemic thinking and the enhancement of systemic competency, (iii) coaching with the system in mind, or taking a (iv) systemic integrated coaching approach, the practical application of the concepts reviewed in this paper have the potential to maximise coaching outcomes for those involved, and those who are connected to those involved.

\section{References}

Allen, J. G., MacNaughton, P., Satish, U., Santanam, S., Vallarino, J., \& Spengler, J. D. (2016). Associations of Cognitive Function Scores with Carbon Dioxide, Ventilation, and Volatile Organic Compound Exposures in Office Workers: A Controlled Exposure Study of Green and Conventional Office Environments. Environmental health perspectives, 124(6), 805-812. 
Anderson, P. (1999). Perspective: Complexity theory and organization science. Organization Science, 10(3), 216-232.

Arnold, R. D., \& Wade, J. P. (2015). A definition of systems thinking: A systems approach. Procedia computer science, 44(1), 669-678.

Bachkirova, T., \& Borrington, S. Beautiful ideas that can make us ill: Implications for coaching. Philosophy of Coaching: An International Journal, 5(1), 9-30. http://dx.doi.org/10.22316/poc/05.1.03.

Bakker, A. B. (2011). An Evidence-Based Model of Work Engagement. Current Directions in Psychological Science, 20(4), 265-269.

Balkundi, P., \& Harrison, D. A. (2006). Ties, leaders, and time in teams: Strong inference about network structure's effects on team viability and performance. Academy of Management journal, 49(1), 49-68.

Balkundi, P., \& Kilduff, M. (2006). The ties that lead: A social network approach to leadership. The leadership quarterly, 17(4), 419-439.

Bono, J. E., \& Anderson, M. H. (2005). The Advice and Influence Networks of Transformational Leaders. Journal of Applied Psychology, 90(6), 13061314.

Bono, J. E., \& Ilies, R. (2006). Charisma, positive emotions and mood contagion. The Leadership Quarterly, 17(4), 317-334.

Borgatti, S. P., Everett, M. G., \& Freeman, L. C. (2014). UCINET.

Bradbury, H., \& Lichtenstein, B. M. (2000). Relationality in organizational research: Exploring the space between.

Brown, C. A. (2006). The application of complex adaptive systems theory to clinical practice in rehabilitation. Disability and rehabilitation, 28(9), 587-593.

Bunker, B. B., \& Alban, B. T. (2012). The handbook of large group methods: Creating systemic change in organizations and communities. John Wiley \& Sons.

Cameron, K. (2012). Positive Leadership: Strategies for Extraordinary Performance: Berrett-Koehler Publishers.

Cameron, K., \& Dutton, J. (2003). Positive Organizational Scholarship: Foundations of a New Discipline. San Francisco: Berrett-Koehler Publishers.

Casciaro, T. (1998). Seeing things clearly: Social structure, personality, and accuracy in social network perception. Social Networks, 20(4), 331-351.

Cavanagh, M. (2006). Coaching from a systemic perspective: A complex adaptive conversation. Evidence Based Coaching Handbook: Putting Best Practice to Work for Your Clients, 313-354. 
Cavanagh, M., \& Lane, D. (2012). Coaching psychology coming of age: The challenges we face in the messy world of complexity. International Coaching Psychology Review, 7(1), 75-90.

Datta, B. (2015). Assessing the Effectiveness of Authentic Leadership. International Journal of Leadership Studies, 9(1), 62-75.

Deci, E. L., Olafsen, A. H., \& Ryan, R. M. (2017). Self-Determination Theory in Work Organizations: The State of a Science. Annual Review of Organizational Psychology and Organizational Behavior, 4, 19-43.

Deci, E. L., \& Ryan, R. M. (2000). The "What" and "Why" of Goal Pursuits: Human Needs and the Self-Determination of Behavior. Psychological Inquiry, 11(4), 227-268.

Dooley, K. J. (1997). A complex adaptive systems model of organization change. Nonlinear dynamics, psychology, and life sciences, 1(1), 69-97.

Dooley, K. J., \& Lichtenstein, B. (2008). Research methods for studying the complexity dynamics of leadership. Complexity Leadership. Part, 1.

Eidelson, R. (1997). Complex adaptive systems in the behavioral and social sciences. Review of General Psychology, 1(1), 42-71.

Fredrickson, B. L. (2000). Cultivating Positive Emotions to Optimize Health and Well-Being. [Article]. Prevention \& Treatment September, 3, 1.

Gagné, M., \& Deci, E. L. (2005). Self - Determination Theory and Work Motivation. Journal of Organizational Behavior, 26(4), 331-362.

Galaskiewicz, J. (2007). Has a Network Theory of Organizational Behaviour Lived Up to its Promises? Management and Organization Review, 3(1), $1-18$.

Gensler, M. (2005). These Four Walls: The Real British Office. London: Gensler.

Grant, A. M. (2013). The efficacy of coaching. Handbook of the psychology of coaching and mentoring, 15-39.

Grant, A. M., O’Connor, S. A., \& Studholme, I. (2019). Towards a Positive Psychology of Buildings and Workplace Community: the Positive Built Workplace Environment. International Journal of Applied Positive Psychology, 4(1-2), 67-89.

Guthrie, J. P. (2001). High-Involvement Work Practices, Turnover, and Productivity: Evidence from New-Zealand. Academy of Management Journal, 44(1), 180-190.

Holland, J. H. (2006). Studying complex adaptive systems. Journal of systems science and complexity, 19(1), 1-8.

Houchin, K., \& MacLean, D. (2005). Complexity theory and strategic change: an empirically informed critique. British Journal of Management, 16(2), 149-166. 
International Coaching Federation \& Human Capital Institution. (2019). Building Strong Coaching Culture for the Future - Final report. NY: USA

Jones, R. J., Woods, S. A., \& Guillaume, Y. R. (2016). The effectiveness of workplace coaching: A meta-analysis of learning and performance outcomes from coaching. Journal of Occupational and Organizational Psychology, 89(2), 249-277.

Kilduff, M., \& Krackhardt, D. (2008). Interpersonal networks in organizations: Cognition, personality, dynamics, and culture (Vol. 30). Cambridge University Press.

Kilduff, M., \& Tsai, W. (2003). Social networks and organizations. Sage.

Krackhardt, D. (1987). Cognitive social structures. Social networks, 9(2), 109134.

Krackhardt, D. (1990). Assessing the political landscape: Structure, cognition, and power in organizations. Administrative science quarterly, 342-369.

Lawrence, P. (2019). What is Systemic Coaching? Philosophy of Coaching: An International Journal. 4(2), 35-52.

MacNaughton, P., Pegues, J., Satish, U., Santanam, S., Spengler, J., \& Allen, J. (2015). Economic, Environmental and Health Implications of Enhanced Ventilation in Office Buildings. International Journal of Environmental Research and Public Health, 12(11), 14,709.

Mann, C. (2019). 6th Ridler Report: Strategic Trends in the Use of Coaching. London:UK.

Mehra, A., Dixon, A. L., Brass, D. J., \& Robertson, B. (2006). The social network ties of group leaders: Implications for group performance and leader reputation. Organization Science, 17(1), 64-79.

Mehra, A., Smith, B. R., Dixon, A. L., \& Robertson, B. (2006). Distributed leadership in teams: The network of leadership perceptions and team performance. Leadership Quarterly, 17(3), 232-245.

Mele, C., Pels, J., \& Polese, F. (2010). A brief review of systems theories and their managerial applications. Service science, 2(1-2), 126-135.

Mulville, M., Callaghan, N., \& Isaac, D. (2016). The impact of the ambient environment and building configuration on occupant productivity in open-plan commercial offices. Journal of Corporate Real Estate.

Novoselich, B. J., \& Knight, D. B. (2018). Shared leadership in capstone design teams: Social network analysis. Journal of Professional Issues in Engineering Education and Practice, 144(4), 04018006.

O'Connor, S., \& Cavanagh, M. (2013). The coaching ripple effect: The effects of developmental coaching on wellbeing across organisational 
networks. Psychology of Well-Being: Theory, Research and Practice, 3(1), 1-23.

O’Connor, S. A., \& Grant, A. M. (2018). Positive psychology meets real estate: The positive built workplace environment. Corporate Real Estate Journal, 8(2), 136-153.

Pierce, J. L., Gardner, D. G., \& Crowley, C. (2016). Organization-based selfesteem and well-being: Empirical examination of a spillover effect. European Journal of Work and Organizational Psychology, 25(2), 181-199.

Rammel, C., Stagl, S., \& Wilfing, H. (2007). Managing complex adaptive systems - a co-evolutionary perspective on natural resource management. Ecological economics, 63(1), 9-21.

Rodan, S. (2010). Structural holes and managerial performance: Identifying the underlying mechanisms. Social Networks, 32(3), 168-179.

Schneider, M., \& Somers, M. (2006). Organizations as complex adaptive systems: Implications of complexity theory for leadership research. The Leadership Quarterly, 17(4), 351-365.

Scott, J. (2000). Social network analysis: A handbook: Sage.

Spence, G. B., \& Deci, E. L. (2013). Self-determination theory within coaching contexts: Supporting motives and goals that promote optimal functioning and well-being. Beyond goals: Effective strategies for coaching and mentoring, 5(11), 85-108.

Spence, G. B., \& Oades, L. G. (2011). Coaching with self-determination theory in mind: Using theory to advance evidence-based coaching practice.

Stacey, R. D. (2001). Complex responsive processes in organizations: Learning and knowledge creation. Psychology Press.

Stacey, R. (2012a). Tools and techniques of leadership and management : meeting the challenge of complexity. Routledge. https://doi.org/10.4324/9780203115893

Stacey, R. (2012b). Comment on debate article: Coaching Psychology Coming of Age: The challenges we face in the messy world of complexity. International Coaching Psychology Review, 7(1), 91-96. http://search.proquest.com/docview/1023096885

Stacey, R. D., \& Mowles, C. (2016). Strategic management and organisational dynamics: The challenge of complexity to ways of thinking about organisations. Pearson education. Harlow, UK.

Terblanche, N. H., \& Erasmus, E. D. (2018). The use of organisational network analysis as a diagnostic tool during team coaching. SA Journal of Industrial Psychology, 44(1), 1-10. 
Tsai, W., \& Ghoshal, S. (1998). Social capital and value creation: The role of intrafirm networks. Academy of Management Journal, 41(4), 464-476.

van Elst, D., \& Meurs, D. (2015). Positive Management: The Relationship between the Psychological Contract, Employee Engagement and Organisational Commitment. Journal of Positive Management, 6(4), 3952.

Von Bertalanffy, L. (1972). The history and status of general systems theory. Academy of management journal, 15(4), 407-426.

Weiner, N. (1948). Cybernetics (1968). MITPress, Cambridge, Massachusetts.

Xie, K., Di Tosto, G., Lu, L., \& Cho, Y. S. (2018). Detecting leadership in peer-moderated online collaborative learning through text mining and social network analysis. The Internet and Higher Education, 38, 9-17.

Zohar, D., \& Tenne-Gazit, O. (2008). Transformational leadership and group interaction as climate antecedents: A social network analysis. Journal of Applied Psychology, 93(4), 744-757

\title{
Acknowledgements
}

I am very grateful and acknowledge the contribution made by the institute of coaching through the Harnisch coaching research grant awarded to partially support the initial research into the coaching ripple effect and also for the funding provided by lend lease, International Towers Sydney that funded the research in to the positive psychology of workplace community and the built environment

\author{
Author Contact \\ Dr Sean O'Connor \\ Director, Coaching Psychology Unit \\ University of Sydney \\ NSW, Australia \\ 2006 \\ E: sean.oconnor@sydney.edu.au
}

